\title{
Performance and Construction of Polar Codes on Symmetric Binary-Input Memoryless Channels
}

\author{
Ryuhei Mori \\ Department of Systems Science \\ Kyoto University \\ Kyoto, 606-8501, Japan \\ Email: rmori@sys.i.kyoto-u.ac.jp
}

\author{
Toshiyuki Tanaka \\ Department of Systems Science \\ Kyoto University \\ Kyoto, 606-8501, Japan \\ Email: tt@i.kyoto-u.ac.jp
}

\begin{abstract}
Channel polarization is a method of constructing capacity achieving codes for symmetric binary-input discrete memoryless channels (B-DMCs) [1]. In the original paper, the construction complexity is exponential in the blocklength. In this paper, a new construction method for arbitrary symmetric binary memoryless channel (B-MC) with linear complexity in the blocklength is proposed. Furthermore, new upper bound and lower bound of the block error probability of polar codes are derived for the BEC and arbitrary symmetric B-MC, respectively.
\end{abstract}

\section{INTRODUCTION}

Channel polarization, introduced by Arkan [1], is a method of constructing capacity achieving codes for symmetric binaryinput discrete memoryless channels (B-DMCs). Polar codes which are realized by channel polarization require only low encoding and decoding complexity for achieving capacity. Furthermore, it was shown by Arıkan and Telatar [2] that the block error probability of polar codes is $O\left(2^{-N^{\beta}}\right)$ for any fixed $\beta<\frac{1}{2}$, where $N$ is the blocklength. It is significantly fast since the block error probability of low-density parity-check (LDPC) codes is polynomial in $N$ [3]. However, in [1], code construction with polynomial complexity is introduced only for the binary erasure channel (BEC). The main result of this paper is to show code construction with $O(N)$ complexity for arbitrary symmetric binary-input memoryless channel (B-MC). Furthermore, a new upper bound and a lower bound of the block error probability of polar codes are derived for the BEC and arbitrary symmetric B-MC, respectively. In Section III channel polarization and polar codes introduced in [1] are described. In Section III, the construction method for arbitrary symmetric B-MC is shown. In Section IV a lower bound of the block error probability of polar codes is derived for arbitrary symmetric B-MC. In Section V a new upper bound of the block error probability of polar codes over the BEC is derived. In Section VI, some techniques for tightening bounds are discussed. In Section VII, numerical calculation results are compared with numerical simulation results. Finally, this paper is concluded in Section VIII

\section{PRELIMINARIES}

\section{A. Channel polarization}

Let the blocklength $N$ be an integer power of 2. In [1], Arikan discussed channel polarization on the basis of an $N \times$
$N$ matrix $G_{N}$, which he called the generator matrix, defined recursively as

$$
G_{2^{n}}:=R_{2^{n}}\left(F \otimes G_{2^{n-1}}\right), \quad G_{2}:=F:=\left[\begin{array}{ll}
1 & 0 \\
1 & 1
\end{array}\right]
$$

where $\otimes$ denotes Kronecker product and where $R_{2^{n}}$ denotes the so-called reverse shuffle matrix, which is a permutation matrix.

For a given B-MC $W:\{0,1\} \rightarrow \mathcal{Y}$, a log-likelihood ratio (LLR) $\log (W(y \mid 0) / W(y \mid 1))$ of $W$ is a sufficient statistic for estimating input $x \in\{0,1\}$ given output $y \in \mathcal{Y}$. Hence, we can associate to $W$ a B-MC $W^{\prime}:\{0,1\} \rightarrow \mathbb{R}$ with the LLR of $W$ as its output, and $W^{\prime}$ has the same performance as $W$ under maximum a posteriori (MAP) decoding. In this paper, we deal with symmetric B-MCs defined as follows.

Definition 1. $A B-M C W:\{0,1\} \rightarrow \mathcal{Y}$ is said to be symmetric if its associated $B-M C W^{\prime}:\{0,1\} \rightarrow \mathbb{R}$ introduced above satisfies $W^{\prime}(y \mid 0)=W^{\prime}(-y \mid 1)$.

Let $I(W)$ denote the capacity between the input and the output of a symmetric B-MC $W$.

We consider communication over a symmetric B-MC $W$ : $\{0,1\} \rightarrow \mathbb{R}$. Let $u_{1}^{N}=\left(u_{1}, u_{2}, \ldots, u_{N}\right)$ denote an $N$ dimensional row vector, and let $u_{i}^{j}=\left(u_{i}, u_{i+1}, \ldots, u_{j}\right)$ be a subvector of $u_{1}^{N}$. Let us consider a vector channel $W_{N}\left(y_{1}^{N}\right.$ $\left.u_{1}^{N}\right):=W^{N}\left(y_{1}^{N} \mid u_{1}^{N} G_{N}\right)$, with input $u_{1}^{N} \in\{0,1\}^{N}$ and output $y_{1}^{N} \in \mathbb{R}^{N}$, which is obtained by combining $N$ parallel B-DMCs $W^{N}\left(y_{1}^{N} \mid x_{1}^{N}\right):=\prod_{i=1}^{N} W\left(y_{i} \mid x_{i}\right)$ via the operation $x_{1}^{N}=u_{1}^{N} G_{N}$, which should be performed in the modulo-2 arithmetic. We define subchannels $W_{N}^{(i)}$ as

$$
W_{N}^{(i)}\left(y_{1}^{N}, u_{1}^{i-1} \mid u_{i}\right):=\frac{1}{2^{N-1}} \sum_{u_{i+1}^{N}} W_{N}\left(y_{1}^{N} \mid u_{1}^{N}\right)
$$

Let $U_{1}^{N} \in\{0,1\}^{N}$ and $Y_{1}^{N} \in \mathbb{R}^{N}$ be random variables which follow the joint probability $W_{N}\left(y_{1}^{N} \mid u_{1}^{N}\right) / 2^{N}$. The mutual information $I\left(U_{1}^{N} ; Y_{1}^{N}\right)$ is split by applying the chain rule, as

$$
\begin{aligned}
I\left(U_{1}^{N} ; Y_{1}^{N}\right) & =\sum_{i=1}^{N} I\left(U_{i} ; Y_{1}^{N} \mid U_{1}^{i-1}\right) \\
& =\sum_{i=1}^{N} I\left(U_{i} ; Y_{1}^{N}, U_{1}^{i-1}\right)-I\left(U_{i} ; U_{1}^{i-1}\right)
\end{aligned}
$$




$$
=\sum_{i=1}^{N} I\left(U_{i} ; Y_{1}^{N}, U_{1}^{i-1}\right)=\sum_{i=1}^{N} I\left(W_{N}^{(i)}\right) .
$$

Arıkan proved the channel polarization property, which states that every term in the last line of (1) takes a value near zero or one, and that since $I\left(U_{1}^{N} ; Y_{1}^{N}\right)=N I(W)$, the approximate numbers of those terms which take values near one and zero are $N I(W)$ and $N(1-I(W))$, respectively. This property suggests the following approach to designing a capacity-achieving error-correcting code: Pick up elements of $u_{1}^{N}$ which correspond to those subchannels with high mutual information $I\left(W_{N}^{(i)}\right)$, and use them as the information bits. Non-information bits in $u_{1}^{N}$ are clamped to prespecified values. The values of the non-information bits are assumed to be allzero in this paper, since they do not affect performance of resulting codes if the transmitting channel is symmetric [1]. Instead of choosing subchannels with high mutual information $I\left(W_{N}^{(i)}\right)$, Arıkan considered another strategy of construction: choosing subchannels with low Bhattacharyya parameters, which is mentioned later in this section.

\section{B. Decoding}

Arıkan considered successive cancellation (SC) decoding in order to achieve capacity with low complexity. In SC decoding, decoding results for the non-information bits are set to 0 . The information bits are decoded sequentially in the ascending order of their indices, via maximum likelihood (ML) decoding of the channel $W_{N}^{(i)}$. More precisely, the decoding result of $i$ th bit is

$$
\hat{U}_{i}\left(y_{1}^{N}, \hat{u}_{1}^{i-1}\right)=\underset{u_{i}=0,1}{\operatorname{argmax}} W_{N}^{(i)}\left(y_{1}^{N}, \hat{u}_{1}^{i-1} \mid u_{i}\right) .
$$

If the two likelihood values are equal, the decoder determines 0 or 1 with probability $1 / 2$.

\section{Upper bound of performance and construction}

When a set $\mathcal{I} \subseteq\{1,2, \ldots, N\}$ of indices of the information bits is fixed, the block error event, denoted by $\mathcal{E}$, of the resulting code with SC decoding is a union over $\mathcal{I}$ of the events $\mathcal{B}_{i, N}$ that the first bit error occurs at the $i$-th bit. One has

$$
\begin{aligned}
\mathcal{B}_{i, N} & =\left\{u_{1}^{N}, y_{1}^{N}, c_{1}^{N} \mid \hat{u}_{1}^{i-1}=u_{1}^{i-1}, \hat{U}_{i}\left(y_{1}^{N}, \hat{u}_{1}^{i-1}\right) \neq u_{i}\right\} \\
& =\left\{u_{1}^{N}, y_{1}^{N}, c_{1}^{N} \mid \hat{u}_{1}^{i-1}=u_{1}^{i-1}, \hat{U}_{i}\left(y_{1}^{N}, u_{1}^{i-1}\right) \neq u_{i}\right\} \\
& \subseteq\left\{u_{1}^{N}, y_{1}^{N}, c_{1}^{N} \mid \hat{U}_{i}\left(y_{1}^{N}, u_{1}^{i-1}\right) \neq u_{i}\right\}=: \mathcal{A}_{i, N}
\end{aligned}
$$

where $c_{1}^{N} \in\{0,1\}^{N}$ denote $N$ independent fair coin flips, with $c_{i}$ being used as the decoding result of $u_{i}$ if the two likelihood values for $u_{i}$ are equal. In [1], $P\left(\mathcal{A}_{i, N}\right)$ is upper bounded by the Bhattacharyya parameter,

$$
Z_{N}^{(i)}:=\sum_{y_{1}^{N}, u_{1}^{i-1}} \sqrt{W_{N}^{(i)}\left(y_{1}^{N}, u_{1}^{i-1} \mid 0\right) W_{N}^{(i)}\left(y_{1}^{N}, u_{1}^{i-1} \mid 1\right)} .
$$

Hence, the block error probability is upper bounded as

$$
P(\mathcal{E})=\sum_{i \in \mathcal{I}} P\left(\mathcal{B}_{i, N}\right) \leq \sum_{i \in \mathcal{I}} P\left(\mathcal{A}_{i, N}\right) \leq \frac{1}{2} \sum_{i \in \mathcal{I}} Z_{N}^{(i)} .
$$

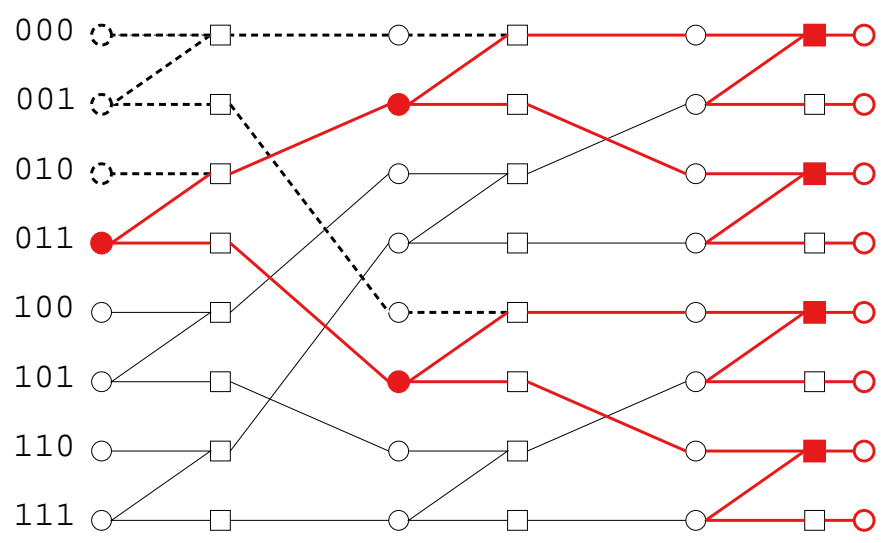

Fig. 1. The decoding tree for $n=3, i=4$. A binary expansion of $(i-1)$ is 011 . Bits 0 and 1 in the expansion correspond to check nodes and variable nodes, which are described as filled squares and filled circles, respectively. Dashed nodes and edges have already been determined to 0 or 1 and thus eliminated. Thin nodes and edges are not useful for decoding for the fourth bit since thin degree- 3 check nodes are connected to a unknown variable node. The leaf nodes are given messages from a channel.

The equality is due to disjointness of $\left\{\mathcal{B}_{i, N}\right\}$. The first inequality follows from the above-mentioned inclusion relation between $\mathcal{A}_{i, N}$ and $\mathcal{B}_{i, N}$. The last inequality is valid for arbitrary symmetric channels [3]. In particular, $Z_{N}^{(i)}=2 P\left(\mathcal{A}_{i, N}\right)$ if and only if the channel is the BEC. Arikan proposed a method of designing a code in which one chooses $\mathcal{I}$ that minimizes the rightmost side of (3), and called the resulting code a polar code. In this paper, we propose an alternative code construction strategy in which $P\left(\mathcal{A}_{i, N}\right)$ is directly evaluated, instead of $Z_{N}^{(i)}$, and $\mathcal{I}$ that minimizes $\sum_{i \in \mathcal{I}} P\left(\mathcal{A}_{i, N}\right)$ is chosen. We call the codes resulting from our strategy polar codes as well.

In the rest of this paper, we use the notations $\mathcal{A}_{i}$ and $\mathcal{B}_{i}$ instead of $\mathcal{A}_{i, N}$ and $\mathcal{B}_{i, N}$, respectively, by dropping the blocklength $N$, when it is evident from the context.

\section{Construction of Polar codes}

We show in this section that $\left\{P\left(\mathcal{A}_{i}\right)\right\}$ are regarded as decoding error probabilities of belief propagation (BP) decoding on tree graphs, so that they can be evaluated via density evolution. The Tanner graph of a polar code for $n=3$ is shown in Fig. 1. Let us consider $i$-th step of SC decoding. Since $u_{1}^{i-1}$ have been either determined as non-information bits or decoded in previous steps, the edges incident to these variable nodes are eliminated. Since $u_{i+1}^{N}$ do not affect the characteristics of the channel $W_{N}^{(i)}$, the degree-3 check nodes connected to them do not work in this stage. Hence, these check nodes and the edges incident to them are eliminated. Similarly, degree-3 check nodes incident to undetermined degree- 1 variable nodes are also eliminated recursively. The resulting decoding graph for $u_{i}$ is tree-like, as shown in Fig. 11 Hence, the ML decision (2) can be implemented by BP decoding on the tree graph. The probability $P\left(\mathcal{A}_{i}\right)$ is therefore regarded as the error probability of the root node of the tree graph via BP decoding, where leaf 
nodes have messages of the channel. Assume that the binary expansion of $(i-1)$ is $b_{n} \ldots b_{1}$, then nodes at depth $t$ of the tree graph are check nodes and variable nodes if $b_{t}=0$ and $b_{t}=1$, respectively, as shown in Fig. 迎.

An LLR for $i$-th bit, defined as $L_{N}^{(i)}\left(y_{1}^{N}, \hat{u}_{i}^{i-1}\right):=$ $\log \left(W_{N}^{(i)}\left(y_{1}^{N}, \hat{u}_{1}^{i-1} \mid 0\right) / W_{N}^{(i)}\left(y_{1}^{N}, \hat{u}_{1}^{i-1} \mid 1\right)\right)$ is calculated recursively as

$$
\begin{aligned}
& L_{N}^{(2 i-1)}\left(y_{1}^{N}, \hat{u}_{1}^{2 i-2}\right) \\
& =2 \tanh ^{-1}\left(\tanh \left(L_{N / 2}^{(i)}\left(y_{1}^{N / 2}, \hat{u}_{1, e}^{2 i-2} \oplus \hat{u}_{1 . o}^{2 i-2}\right) / 2\right)\right. \\
& \left.\quad \times \tanh \left(L_{N / 2}^{(i)}\left(y_{N / 2+1}^{N}, \hat{u}_{1, e}^{2 i-2}\right) / 2\right)\right) \\
& L_{N}^{(2 i)}\left(y_{1}^{N}, \hat{u}_{1}^{2 i-1}\right)=L_{N / 2}^{(i)}\left(y_{N / 2+1}^{N}, \hat{u}_{1, e}^{2 i-2}\right) \\
& +(-1)^{\hat{u}_{2 i-1}} L_{N / 2}^{(i)}\left(y_{1}^{N / 2}, \hat{u}_{1, e}^{2 i-2} \oplus \hat{u}_{1, o}^{2 i-2}\right)
\end{aligned}
$$

where $\hat{u}_{1, e}^{i}$ and $\hat{u}_{1, o}^{i}$ denote subvectors which consist of elements of $\hat{u}_{1}^{i}$ with even and odd indices, respectively, and where $\oplus$ denotes modulo- 2 addition. The above updating rules are originally derived by Arıkan [1].

It is well known in the field of LDPC codes that the error probability of the root node of a tree graph after message passing decoding is calculated via density evolution. For the analysis of the error probability of symmetric channels, without loss of generality, it is assumed that the all-zero message is transmitted. The following theorem for symmetric $\mathrm{B}-\mathrm{MC}$ is a consequence of a well-known result in [3] and also obtained for the BEC by Arıkan [1].

Theorem 1. For a symmetric B-MC which has a density $\mathrm{a}_{W}$ of LLR, it holds that $P\left(\mathcal{A}_{i}\right)=\mathfrak{E}\left(\mathrm{a}_{N}^{i}\right)$ where

$$
\begin{gathered}
\mathfrak{E}(\mathrm{a}):=\lim _{\epsilon \rightarrow+0}\left(\int_{-\infty}^{-\epsilon} \mathrm{a}(x) \mathrm{d} x+\frac{1}{2} \int_{-\epsilon}^{+\epsilon} \mathrm{a}(x) \mathrm{d} x\right), \\
\mathrm{a}_{2 N}^{2 i}=\mathrm{a}_{N}^{i} \star \mathrm{a}_{N}^{i}, \quad \mathrm{a}_{2 N}^{2 i-1}=\mathrm{a}_{N}^{i} \text { 因 } \mathrm{a}_{N}^{i}, \quad \mathrm{a}_{1}^{1}=\mathrm{a}_{W}
\end{gathered}
$$

and where $\star$ and $⿴$ denote the convolutions of LLR density functions, which are defined in [3], corresponding to variable and check nodes, respectively.

On the basis of the availability of $P\left(\mathcal{A}_{i}\right)$ s assured by Theorem 1, we propose the following code construction procedure: Choose $\mathcal{I}$ which minimizes

$$
\sum_{i \in \mathcal{I}} P\left(\mathcal{A}_{i}\right)
$$

subject to $|\mathcal{I}|=N R$. The block error probability of the resulting codes also decays like $O\left(2^{-N^{\beta}}\right)$ for any $\beta<\frac{1}{2}$ as in [2], since the upper bound of the block error probability, given in terms of Bhattacharyya parameters in [2], is also an upper bound of the block error probability of the codes constructed via the proposed method.

In [1], complexity of code construction on the BEC is explained as $O(N \log N)$. However, Theorem 11 states that

\footnotetext{
${ }^{1}$ In counting the depth we omit nodes in the tree with degree 2 , because messages of BP are passed through such nodes unprocessed.
}

the complexity of code construction, not only for the BEC but also for an arbitrary symmetric B-MC, is $O(N)$. To see this, let $\chi(N)$ denote the complexity of calculation of $\left\{a_{N}^{i}\right\}_{i=1, \ldots, N}$ where the complexities of computations of $\star$ and $⿴$ are considered to be constant. Then, it is evaluated as

$$
\chi(N)=N+\chi\left(\frac{N}{2}\right)=N+\frac{N}{2}+\frac{N}{4}+\cdots+1=O(N) .
$$

Since the complexity of selecting the $N R$-th smallest $P\left(\mathcal{A}_{i}\right)$ is $O(N)$ even in the worst case [4], the complexity of code construction is $O(N)$.

We would like to note that larger $N$ requires higherprecision representation of messages for reliable SC decoding and density evolution computations. In this regard, the complexity of SC decoding discussed in [1] and the complexity of construction mentioned above should be understood as referring to the number of the arithmetics of LLRs in BP and the number of the convolution operations in density evolution, respectively, not mentioning their precision. In practice, use of finite-sized binning in density evolution may lead to imprecise upper bounds of the block error probabilities, which, however, still provide upper bounds relevant to SC decoding with the same quantization as the binning scheme.

\section{LOWER BOUND OF THE BLOCK ERROR PROBABILITY FOR ARBITRARY SYMMETRIC B-MC}

To the authors' knowledge, no lower bound of the block error probability of polar codes has been known. In this section, we introduce a lower bound for a given choice of information bits $\mathcal{I}$. We use the following fundamental lemma.

Lemma 1. $\bigcup_{i \in \mathcal{I}} \mathcal{B}_{i}=\bigcup_{i \in \mathcal{I}} \mathcal{A}_{i}$.

Proof: The direction $\subseteq$ is trivial. Assume an event $v$ belongs to $\mathcal{A}_{i}$. If $\hat{u}_{1}^{i-1}=u_{1}^{i-1}, v$ belongs to $\mathcal{B}_{i}$. Otherwise, $v$ belongs to $\mathcal{B}_{j}$ for some $j<i$ which belongs to $\mathcal{I}$.

Recalling $\mathcal{E}=\bigcup_{i \in \mathcal{I}} \mathcal{B}_{i}$, it immediately follows that $P(\mathcal{E})=$ $P\left(\bigcup_{i \in \mathcal{I}} \mathcal{A}_{i}\right)$ holds. The events $\left\{\mathcal{A}_{i}\right\}$ are easier to deal with than $\left\{\mathcal{B}_{i}\right\}$. Several bounds which use probabilities concerning $\left\{\mathcal{A}_{i}\right\}$ are considered in what follows.

First, via Boole's inequality, the following lower bound is obtained for any $\mathcal{S} \subseteq \mathcal{I}$

$$
\begin{aligned}
P\left(\bigcup_{i \in \mathcal{I}} \mathcal{A}_{i}\right) & \geq P\left(\bigcup_{i \in \mathcal{S}} \mathcal{A}_{i}\right) \\
& \geq \sum_{i \in \mathcal{S}} P\left(\mathcal{A}_{i}\right)-\sum_{(i, j) \in \mathcal{S}^{2}, i<j} P\left(\mathcal{A}_{i} \cap \mathcal{A}_{j}\right) .
\end{aligned}
$$

Maximization of the lower bound (5) with respect to $\mathcal{S}$ is difficult since it is equivalent to the Max-Cut problem, which is NP-hard [5]. However, without strict optimization, one can obtain practically accurate lower bounds for some rates and channels.

In order to obtain the lower bound (5), evaluations of probabilities of intersections of two $\mathcal{A}_{i} \mathrm{~s}$ are required. For this purpose, we introduce a method which we call the joint density evolution. Let $\left(X_{1}, Y_{1}\right)$ and $\left(X_{2}, Y_{2}\right)$ denote pairs 
of random variables which independently follow $\mathrm{a}(x, y)$ and $\mathrm{b}(x, y)$, respectively. The convolution $\mathrm{a} \star \star \mathrm{b}$ is defined as the joint density function of messages $(X, Y)$ where $X=X_{1}+X_{2}$ and $Y=Y_{1}+Y_{2}$. Similarly, the convolutions $\mathrm{a} \star \circledast ㇒ \mathrm{~b}$ is defined as the joint density function of messages $(X, Y)$ where $X=X_{1}+X_{2}$ and $Y=2 \tanh ^{-1}\left(\tanh \left(Y_{1} / 2\right) \tanh \left(Y_{2} / 2\right)\right)$. The other convolutions $\mathrm{a} * \star \mathrm{b}$ and $\mathrm{a}$ 国困 $\mathrm{b}$ are also defined in the same way.

Theorem 2. For a symmetric B-MC which has a density $\mathrm{a}_{W}$ of LLR, the joint density $a_{N}^{i, j}$ of LLR for $i$-th bit and $j$-th bit after BP decoding is calculated recursively as

$$
\begin{aligned}
& a_{2 N}^{2 i, 2 j}=a_{N}^{i, j} \star \star a_{N}^{i, j}, \quad a_{2 N}^{2 i, 2 j-1}=a_{N}^{i, j} \star \circledast a_{N}^{i, j}, \\
& \mathrm{a}_{2 N}^{2 i-1,2 j}=\mathrm{a}_{N}^{i, j} \circledast \star \mathrm{a}_{N}^{i, j}, \quad \mathrm{a}_{2 N}^{2 i-1,2 j-1}=\mathrm{a}_{N}^{i, j} \text { 目困 } \mathrm{a}_{N}^{i, j}, \\
& \mathrm{a}_{1}^{1}(x, y)=\delta(x-y) \mathrm{a}_{W}(x)
\end{aligned}
$$

where $\delta(x)$ denotes the Dirac delta function.

The probabilities $P\left(\mathcal{A}_{i} \cap \mathcal{A}_{j}\right), P\left(\mathcal{A}_{i}^{c} \cap \mathcal{A}_{j}\right), P\left(\mathcal{A}_{i} \cap \mathcal{A}_{j}^{c}\right)$ and $P\left(\mathcal{A}_{i}^{c} \cap \mathcal{A}_{j}^{c}\right)$ are calculated by appropriate integrations of the joint density $a_{N}^{i, j}$. Extensions of joint density evolution to higher order joint distributions are also possible straightforwardly.

For the BEC, density evolution has only to evolve expectations of erasure probabilities [3]. Correspondingly, joint density evolution for the BEC is much simpler than that for a general symmetric B-MC, as follows.

Corollary 1. For the BEC with erasure probability $\epsilon$,

$$
\begin{aligned}
a_{N}^{i, j}(x, y) & =p_{N}^{i, j}(0,0) \delta(x) \delta(y)+p_{N}^{i, j}(0,1) \delta(x) \delta_{\infty}(y) \\
& +p_{N}^{i, j}(1,0) \delta_{\infty}(x) \delta(y)+p_{N}^{i, j}(1,1) \delta_{\infty}(x) \delta_{\infty}(y)
\end{aligned}
$$

where

$$
\begin{aligned}
p_{2 N}^{2 i, 2 j}(0,0)= & p_{N}^{i, j}(0,0)^{2}, \\
p_{2 N}^{2 i, 2 j}(0,1)= & p_{N}^{i, j}(0,1)^{2}+2 p_{N}^{i, j}(0,0) p_{N}^{i, j}(0,1), \\
p_{2 N}^{2 i, 2 j}(1,0)= & p_{N}^{i, j}(1,0)^{2}+2 p_{N}^{i, j}(0,0) p_{N}^{i, j}(1,0), \\
p_{2 N}^{2 i, 2 j}(1,1)= & 1-p_{2 N}^{2 i, 2 j}(0,0) \\
& -p_{2 N}^{2 i, 2 j}(0,1)-p_{2 N}^{2 i, 2 j}(1,0), \\
p_{2 N}^{2 i, 2 j-1}(0,0)= & p_{N}^{i, j}(0,0)^{2}+2 p_{N}^{i, j}(0,0) p_{N}^{i, j}(0,1), \\
p_{2 N}^{2 i, 2 j-1}(0,1)= & p_{N}^{i, j}(0,1)^{2}, \\
p_{2 N}^{2 i, 2 j-1}(1,1)= & p_{N}^{i, j}(1,1)^{2}+2 p_{N}^{i, j}(1,1) p_{N}^{i, j}(0,1), \\
p_{2 N}^{2 i, 2 j-1}(1,0)= & 1-p_{2 N}^{2 i, 2 j-1}(0,0) \\
& -p_{2 N}^{2 i, 2 j-1}(0,1)-p_{2 N}^{2 i, 2 j-1}(1,1), \\
p_{2 N}^{2 i-1,2 j}(0,0)= & p_{N}^{i, j}(0,0)^{2}+2 p_{N}^{i, j}(0,0) p_{N}^{i, j}(1,0), \\
p_{2 N}^{2 i-1,2 j}(1,0)= & p_{N}^{i, j}(1,0)^{2}, \\
p_{2 N}^{2 i-1,2 j}(1,1)= & p_{N}^{i, j}(1,1)^{2}+2 p_{N}^{i, j}(1,1) p_{N}^{i, j}(1,0), \\
p_{2 N}^{2 i-1,2 j}(0,1)= & 1-p_{2 N}^{2 i-1,2 j}(0,0) \\
& -p_{2 N}^{2 i-1,2 j}(1,0)-p_{2 N}^{2 i-1,2 j}(1,1),
\end{aligned}
$$

$$
\begin{aligned}
p_{2 N}^{2 i-1,2 j-1}(0,1)= & p_{N}^{i, j}(0,1)^{2}+2 p_{N}^{i, j}(0,1) p_{N}^{i, j}(1,1), \\
p_{2 N}^{2 i-1,2 j-1}(1,0)= & p_{N}^{i, j}(1,0)^{2}+2 p_{N}^{i, j}(1,0) p_{N}^{i, j}(1,1), \\
p_{2 N}^{2 i-1,2 j-1}(1,1)= & p_{N}^{i, j}(1,1)^{2}, \\
p_{2 N}^{2 i-1,2 j-1}(0,0)= & 1-p_{2 N}^{2 i-1,2 j-1}(0,1) \\
& -p_{2 N}^{2 i-1,2 j-1}(1,0)-p_{2 N}^{2 i-1,2 j-1}(1,1), \\
p_{1}^{1,1}(0,0)= & \epsilon, \quad p_{1}^{1,1}(0,1)=0, \\
p_{1}^{1,1}(1,0)= & 0, \quad p_{1}^{1,1}(1,1)=1-\epsilon
\end{aligned}
$$

and where $\delta_{\infty}(x)$ denotes the Dirac delta function of unit mass at infinity.

Higher-order joint probabilities such as $P\left(\mathcal{A}_{i} \cap \mathcal{A}_{j} \cap \mathcal{A}_{k}\right)$ are calculated recursively by tracking real vectors of an appropriate dimension in a way similar to that described in Corollary 1

Complexity of computations (as measured in numbers of convolution operations) of all $\mathrm{a}_{N}^{i, j} \mathrm{~s}$ is $O\left(N^{2}\right)$ as $N$ increases. Similarly, complexity of computations of all $s$-joint densities $\mathrm{a}_{N}^{i_{1}, \ldots, i_{s}} \mathrm{~s}$ is $O\left(N^{s}\right)$ as $N$ increases. On the other hand, the complexity grows exponentially in $s$ since the dimension of the densities is $s$.

\section{NEW UPPER BOUND OF THE BLOCK ERROR PROBABILITY FOR THE BEC}

The upper bound (4) of the block error probability of polar codes may yield poor results. In particular, it exceeds one near the capacity, as observed in [1] for the BEC. In this section, a new upper bound which does not exceed one is derived for the BEC. For the BEC, covariances among complements of $\left\{\mathcal{A}_{i}\right\}$, denoted by $\left\{\mathcal{A}_{i}^{c}\right\}$, are always positive.

Lemma 2. For the BEC, $P\left(\bigcap_{i \in \mathcal{I}} \mathcal{A}_{i}^{c}\right) \geq \prod_{i \in \mathcal{I}} P\left(\mathcal{A}_{i}^{c}\right)$, for any $\mathcal{I} \subseteq\{1, \ldots, N\}$.

Outline of proof: An event $\mathcal{A}_{i}^{c}$ is expressed as $\bigcap_{k}\left\{e_{i, k} \nsubseteq \mathcal{F}\right\}$, where $e_{i, k}$ is an error pattern of erasure messages for $i$-th bit, and where $\mathcal{F}$ is a set of indices of erasure messages.

Using this property, the block error probability is upper bounded simply by $1-\prod_{i \in \mathcal{I}} P\left(\mathcal{A}_{i}^{c}\right)$. Furthermore, it is more accurately upper bounded by

$$
1-\prod_{i \in \mathcal{I}} P\left(\mathcal{A}_{i}^{c} \mid \mathcal{A}_{p(i)}^{c}\right)
$$

where $p(i) \in \mathcal{I}$ corresponds to a parent node of $\mathcal{A}_{i}$ in a spanning tree of a perfect graph which has nodes corresponding to indices in $\mathcal{I} . P\left(\mathcal{A}_{i}^{c} \mid \mathcal{A}_{p(i)}^{c}\right)$ is calculated via joint density evolution. In order to tighten the upper bound (6), the maximum weight directed spanning tree should be chosen from the perfect directed graph whose edges have weights $P\left(\mathcal{A}_{i}^{c} \mid \mathcal{A}_{j}^{c}\right)$, where $i$ and $j$ are sink and source nodes of the directed edge, respectively, like Chow-Liu tree [6]. 


\section{TEChNiques FOR TIGHTENING BOUNDS}

In this section, some techniques for obtaining tighter bounds of $P\left(\bigcup_{i \in \mathcal{I}} A_{i}\right)$ are shown. The first one is applicable to polar codes over the BEC. In this case, the LLR $L_{N}^{(i)}\left(y_{1}^{N}, \hat{u}_{j}^{i-1}\right)$ for $i$-th bit in SC decoding, when the all-zero information is transmitted, is either zero or infinity. Let $\mathcal{A}_{i}^{\prime}$ be the event that the LLR for $i$-th bit is zero. We consider the events of erasure $\left\{\mathcal{A}_{i}^{\prime}\right\}$ rather than $\left\{\mathcal{A}_{i}\right\}$ for simplicity. We first define partial ordering on $\left\{1, \ldots, 2^{n}\right\}$.

Definition 2. For $i, j \in\left\{1, \ldots, 2^{n}\right\}, i \prec j$ if and only if $t$-th bit of binary expansion of $j-1$ is one when $t$-th bit of binary expansion of $i-1$ is one for any $t \in\{1, \ldots, n\}$.

The following theorem is useful for reducing time complexity of calculations of the bounds.

Lemma 3. $j \prec i \Longleftrightarrow \mathcal{A}_{i}^{\prime} \subseteq \mathcal{A}_{j}^{\prime}$.

Proof: If a variable node outputs an erased message, a check node with the same input as the variable node outputs an erased message. Hence if $v \in \mathcal{A}_{i}^{\prime}$ then $v \in \mathcal{A}_{j}^{\prime}$. The proof of the other direction is also obvious and is omitted.

Theorem 3. The block erasure probability of polar codes of information bits $\mathcal{I}$ is $P\left(\bigcup_{i \in M(\mathcal{I})} \mathcal{A}_{i}^{\prime}\right)$ where $M(\mathcal{I})$ denotes the set of minimal elements of $\mathcal{I}$ with respect to $\prec$.

Proof: From Lemma 3, $\bigcup_{i \in \mathcal{I}} \mathcal{A}_{i}^{\prime}=\bigcup_{i \in M(\mathcal{I})} \mathcal{A}_{i}^{\prime}$. From this Theorem, we have only to consider the set of minimal elements $M(\mathcal{I})$ for the block erasure probability of polar codes over the BEC, which can be used to tighten bounds.

For polar codes over a general symmetric B-MC, the following result similar to Theorem 3 is obtained.

Theorem 4. For integers $0 \leq k \leq n$, and $0 \leq i \leq 2^{n-k}-1$ $P\left(\mathcal{A}_{2^{k} i+1,2^{n}} \cup \cdots \cup \mathcal{A}_{2^{k}(i+1), 2^{n}}\right)=1-\left(1-P\left(\mathcal{A}_{i+1,2^{n-k}}\right)\right)^{2^{k}}$

Proof is omitted for lack of space. Although the joint probability $P\left(\mathcal{A}_{2^{k} i+1,2^{n}} \cup \cdots \cup \mathcal{A}_{2^{k}(i+1), 2^{n}}\right)$ can be calculated via joint density evolution, Theorem 4 allows us to calculate it more efficiently via density evolution for depth- $(n-k)$ trees and a few arithmetics.

From Theorem 4 one can efficiently obtain a tighter upper bound than (4) by decomposing the block error event as $\mathcal{E}=\bigcup_{i \in \mathcal{I}} \mathcal{A}_{i}=\bigcup_{j \in \mathcal{J}} \mathcal{C}_{j}$, where each $\mathcal{C}_{j}$ is expressed as $\mathcal{A}_{2^{k} i+1,2^{n}} \cup \cdots \cup \mathcal{A}_{2^{k}(i+1), 2^{n}}$. For example, if we choose $\mathcal{I}=$ $\{4,6,7,8\}$ as information bits for a polar code with $N=8$, one obtains an upper bound $P\left(\mathcal{A}_{4}\right)+P\left(\mathcal{A}_{6}\right)+P\left(\mathcal{A}_{7} \cup \mathcal{A}_{8}\right)$, which is tighter than $P\left(\mathcal{A}_{4}\right)+P\left(\mathcal{A}_{6}\right)+P\left(\mathcal{A}_{7}\right)+P\left(\mathcal{A}_{8}\right)$.

\section{Numerical CALCUlations AND SIMUlations}

In this section, numerical calculation results are compared with numerical simulation results. Figure 2 shows calculation results of the upper bounds (4), (6) and the lower bound (5) of block erasure probability. Coding rate is 0.5 and blocklength is 1024. Only the minimal elements of information bits are considered in view of Theorem 3 for calculation of these

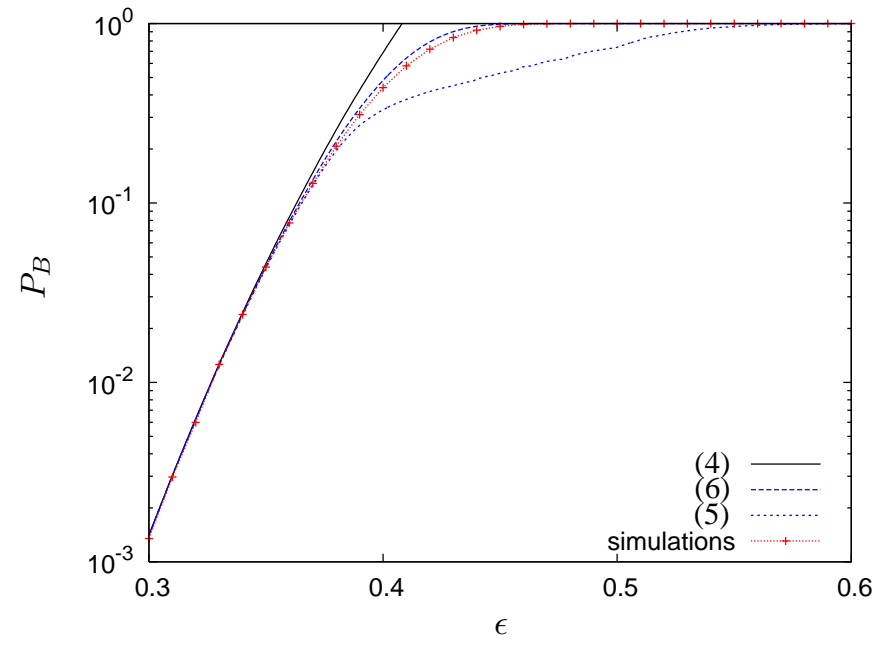

Fig. 2. Calculation results of upper bounds (4), (6) and lower bound (5) Rate is 0.5. Blocklength is 1024 .

bounds. Although we optimized the upper bound (6) and the lower bound (5) only approximately, the lower bound is very close to the upper bound for $\epsilon$ below 0.4. Our new upper bound is always smaller than 1 and closer to the simulation results, whereas the union bound exceeds 1 when $\epsilon>0.407$.

\section{CONCLUSION AND FUTURE WORKS}

The construction method of polar codes for symmetric BMCs with complexity $O(N)$ is shown. New upper and lower bounds for the block error probability of particular polar codes and the method of joint density evolution are derived. The method and the bounds are also applicable to generalized polar codes [7].

Computing higher-order joint distributions and deriving other bounds (e.g., Boole's inequality with higher-order terms) are future works.

\section{ACKNOWLEDGMENT}

TT acknowledges support of the Grant-in-Aid for Scientific Research on Priority Areas (No. 18079010), MEXT, Japan.

\section{REFERENCES}

[1] E. Arıkan, "Channel polarization: A method for constructing capacityachieving codes for symmetric binary-input memoryless channels," 2008. [Online]. Available: http://arxiv.org/abs/0807.3917

[2] E. Arrkan and E. Telatar, "On the rate of channel polarization," 2008. [Online]. Available: http://arxiv.org/abs/0807.3806

[3] T. Richardson and R. Urbanke, Modern Coding Theory. Cambridge University Press, 2008.

[4] M. Blüm, R. Floyd, V. Pratt, R. Rivest, and R. Tarjan, "Time bounds for selection," Journal of Computer and System Sciences, vol. 7, pp. 448-461, 1973.

[5] A. Caprara, "Constrained 0-1 quadratic programming: Basic approaches and extensions," European Journal of Operational Research, vol. 187, no. 3, pp. 1494-1503, 2008.

[6] C. Chow and C. Liu, "Approximating discrete probability distributions with dependence trees," IEEE Trans. Inf. Theory, vol. 14, no. 3, pp. 462 467, 1968.

[7] S. Korada, E. Şaşoğlu, and R. Urbanke, "Polar codes: characterization of exponent, bounds, and constructions," 2009. [Online]. Available: http://arxiv.org/abs/0901.0536 